\title{
Xanthoceraside prevented synaptic loss and reversed learning-memory deficits in APP/PS1 transgenic mice
}

\author{
Ge Jin ${ }^{1,2} \cdot$ Lin Zhu $^{1,2} \cdot$ Peng Liu ${ }^{1} \cdot$ Qian Xu ${ }^{1} \cdot$ Yue Qi ${ }^{1} \cdot$ Xiaoyu Zhou ${ }^{1} \cdot$ Jikai Xu $^{1} \cdot$ Xuefei $\mathrm{Ji}^{1} \cdot$ Tianyan Chi $^{1} \cdot$ Libo Zou $^{1}$
}

Received: 10 September 2018 / Accepted: 22 January 2019 / Published online: 14 February 2019

(C) The Physiological Society of Japan 2019

\begin{abstract}
Xanthoceraside, a novel triterpenoid saponin, has been found to attenuate learning and memory impairments in AD animal models. However, whether xanthoceraside has a positive effect on synaptic morphology remains unclear. Herein, we evaluated the effects of xanthoceraside on learning and memory impairments and the abnormalities of synaptic structure in APP/PS1 transgenic mice. The behavioral experiments demonstrated that xanthoceraside attenuated the imaginal memory and spatial learning impairments, and improved social interaction. Transmission electron microscopy and Golgi staining showed that xanthoceraside ameliorated synapse morphology abnormalities and dendritic spine density deficits, respectively. Western-blot analysis identified that xanthoceraside increased the expression of SYP and PSD95, activated BDNF/TrkB/MAPK/ERK and PI3K/Akt signaling pathways, meanwhile decreased the expression of RhoA, ROCK and Snk, increased the levels of SPAR, and activated the BDNF/TrkB/cofilin signaling pathway. Taken together, our study indicated that xanthoceraside improved cognitive function and protected both synaptic morphology and dendritic spine in APP/PS1 transgenic mice, which might be related in part to its activation in the BDNF/TrkB pathway.
\end{abstract}

Keywords Xanthoceraside $\cdot$ Learning and memory $\cdot$ Synaptic structure $\cdot$ BDNF $\cdot$ TrkB

$\begin{array}{ll}\text { Abbreviations } \\ \text { A } \beta & \text { Amyloid- } \beta \text { peptide } \\ \text { NFTs } & \text { Intracellular neurofibrillary tangles } \\ \text { BDNF } & \text { Brain-derived neurotrophic factor } \\ \text { TrkB } & \text { Tropomyosin receptor kinase B } \\ \text { MAPK } & \text { Mitogen-activated protein kinases } \\ \text { ERK } & \text { Extracellular-signal related kinase }\end{array}$

Ge Jin and Lin Zhu contributed equally to this study and should be regarded as co-first authors.

\author{
PI3K Phosphatidylinositol 3 kinase \\ RhoA Ras homolog gene A \\ ROCK2 Rho-associated coiled-coil forming protein \\ kinase 2 \\ Snk Serum inducible kinase \\ SPAR Spine-associated Rap-specific GTPase-activat- \\ ing protein \\ SYP Synaptophysin \\ PSD95 Post-synaptic density protein
}

Jikai Xu

2525252525@163.com

Xuefei Ji

107953319@qq.com

Tianyan Chi

chitianyan@163.com

1 Department of Pharmacology, Life Science and Biopharmaceutics School, Shenyang Pharmaceutical University, 103 Wenhua Road, Shenhe District, Shenyang 110016, Liaoning, People's Republic of China

2 Shenyang Medical College, Shenyang 110034, People's Republic of China

Xiaoyu Zhou

1205970774@qq.com 


\section{Introduction}

Alzheimer's disease (AD) is an age-related progressive neurodegenerative disease. Amyloid- $\beta$ peptide (A $\beta)$ deposition induced senile plaque, intracellular neurofibrillary tangles (NFTs), and loss of neurons and synapses in the brain are the primary pathological changes of AD [1]. Synapse formation plays a vital role in the process of brain development, and synaptic abnormalities, which lead to dysfunction in brain neural circuitry. Dystrophic neurites, found around senile plaque, are impaired neurons that contain the abnormal cytoskeletal elements and accumulated proteins, the same as found in neurofibrillary tangles [2-4]. In typical AD impairment, progressive cognitive and emotional disturbances are the result of synaptic structural and functional abnormalities and neuronal death in the hippocampus and cerebral cortex [5-7]. The literature has reported that soluble $A \beta$ oligomers damage synaptic plasma membranes and that accumulated tau induces significant synaptic degeneration $[8$, 9]. These studies indicate that soluble, oligomeric $A \beta$ and tau are two major factors of synaptotoxicities $[10,11]$. Additionally, several studies found that dendrites and dendritic spines near amyloid plaques suffer severer damage in APP/PS1 transgenic mice, revealing the spatial correlation between $\mathrm{A} \beta$, plaques, and synaptic abnormalities [12, 13]. Therefore, synaptic plasticity impairment could be related to $\mathrm{AD}$ progression.

Brain-derived neurotrophic factor (BDNF) binds to its receptor, tropomyosin receptor kinase $\mathrm{B}$ (TrkB), to further trigger many signaling molecules, including mitogen-activated protein kinases (MAPK)/extracellular-signal related kinase (ERK), and the phosphatidylinositol 3 kinase (PI3K)/Akt pathways [14]. Besides BDNF, some growth factors, such as VEGF [15, 16] and bFGF [17, 18], also regulate the ERK and Akt pathways. BDNF contributes to synapse development, activity, and stability, and facilitates the formation of hippocampus-dependent memory [19]. BDNF deficiency leads to synapse injury, neuron apoptosis, and cholinergic neurotransmission dysfunction [20].

Ras homolog gene A (RhoA) and Rho-associated coiled-coil forming protein kinase 2 (ROCK2) are two important mediators to block central nervous cell regeneration. Genetic mutations in Rho-GTPase-dependent pathways result in severe cognitive deficits [21]. A recent study showed that RhoA is converted into its active state from an inactive state in response to extracellular signals, which passes to the downstream of ROCK. Then, ROCK acts on actin-myosin, leading to cytoskeleton collapse and neurite growth inhibition [22]. Serum inducible kinase (Snk) phosphorylates spine-associated Rapspecific GTPase-activating protein (SPAR) and leads to its degradation. Depletion of SPAR from dendritic spines leads to spine shrinkage and loss [23]. BDNF/TrkB receptors are expressed on the membranes to control the actin cytoskeleton in dendritic spines, as well as to regulate the formation and regression of dendritic spines [24]. Cofilin is an essential protein that enhances the rapid disassembly of actin, which regulates neuronal structure. BDNF acts on the TrkB receptor to activate Ras-related $\mathrm{C} 3$ botulinum toxin substrate 1 (Rac1) and enhance the downstream target of Rac1-Rho kinase/p21-activated kinase (PAK), and finally inhibit cofilin upon depolymerization of F-actin to maintain the structure of dendritic spines [25]. A study shows that the treatment with BDNF on hippocampal slices from rodents facilitates the long-term potentiation and synaptic growth, increases the dendrite spine density in the hippocampus CA1 region, and the expression of synaptic proteins [26]. The levels of BDNF mRNA and protein are decreased in several cognition-associated brain areas (like hippocampus and parietal cortex) of AD animal models and AD patients, which correlated with clinical neuropsychological scores [27]. BDNF could protect the $A \beta$-induced neurotoxicity in vivo and in vitro [28]. Therefore, the function of BDNF might be related to the formation of synapse and memory.

Xanthoceras sorbifolia Bunge is a solo-type Xanthoceras belonging to the Sapindaceae family, which is distributed widely in northern China and Mongolia. It has been used to treat inflammation, enuresis, and rheumatism in Chinese and Mongolian traditional medicine. Xanthoceraside [29], a triterpenoid saponin, is extracted from the husk of Xanthoceras sorbifolia Bunge. Our previous studies show that xanthoceraside prevented $\mathrm{A} \beta_{25-35}$ and $\mathrm{A} \beta_{1-42}$-induced cognitive disorders in rodents [30]. Xanthoceraside also inhibits tau hyper-phosphorylation in the hippocampus of ICV-streptozotocin-induced AD rats, and regulates microglial activation in primary cultured rat microglia and $\mathrm{N} 9$ microglia cell lines $[31,32]$. However, whether xanthoceraside prevents synapse dysfunction remains unclear. In this study, we firstly evaluated the effect of xanthoceraside on cognitive deficits in APP/PS1 transgenic mice. We also assessed the protective effect of xanthoceraside on synaptic morphology in the hippocampus of APP/PS1 transgenic mice and its potential mechanism.

\section{Materials and methods}

\section{Drugs}

Xanthoceraside was provided by the Department of Natural Products Chemistry, Shenyang Pharmaceutical University (Shenyang, China) and dissolved in double-distilled water (0.1\% DMSO). The structure of Xanthoceraside is shown 
Fig. 1 Chemical structure of xanthoceraside

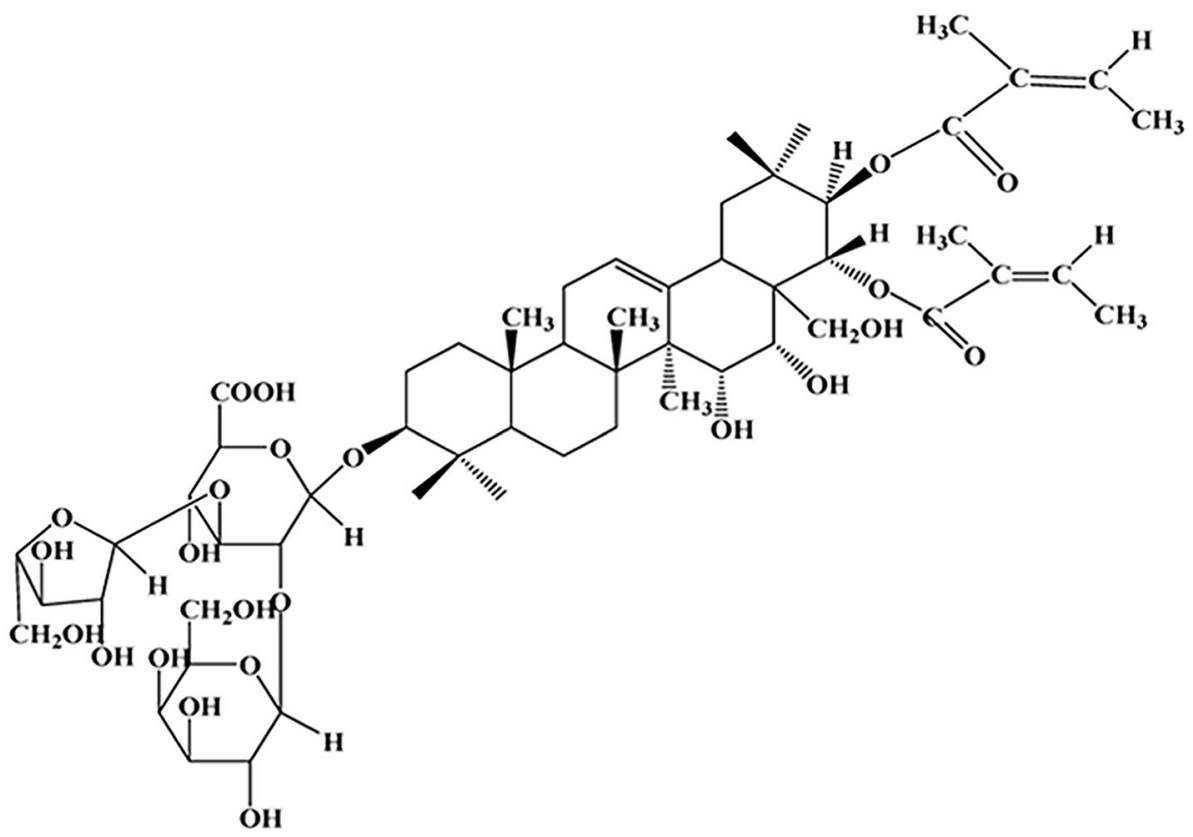

in Fig. 1. Donepezil hydrochloride was procured from Eisai (Japan).

\section{Animals and drug administration}

Male APPswe/PS1 ${ }_{\triangle \mathrm{E} 9}(\mathrm{APP} / \mathrm{PS} 1)$ transgenic mice with a C57BL/6 background were both procured from the HuaFuKang (HFK) Bioscience Co. Ltd. The animals were kept in a polyacrylic cage $(34 \times 22 \times 15 \mathrm{~cm})$ and maintained under standard housing conditions (room temperature $22.5 \pm 1{ }^{\circ} \mathrm{C}$ and humidity $50 \pm 2 \%$ ) with a 12 -h light/dark cycle. Food and water were available ad libitum. Mice were randomly divided into model, donepezil, and xanthoceraside treatment groups. Xanthoceraside $(0.02,0.08$, or $0.32 \mathrm{mg} / \mathrm{kg}$ ) or donepezil $(1.3 \mathrm{mg} / \mathrm{kg})$ were administered orally by gavage, whereas model group mice and $\mathrm{C} 57 \mathrm{BL} / 6 \mathrm{~J}$ mice were treated with a vehicle. APP/PS1 transgenic mice at 7 months of age were orally treated with xanthoceradide for 3 months. Male C57BL/6J mice at the same age were treated with a vehicle as negative controls. The mice were treated once daily or $1 \mathrm{~h}$ before the behavioral tests.

\section{Novel object recognition test}

The novel object recognition test was performed per our previous report [30]. Briefly, after 3 months of drug administration, each mouse was placed into a square box $(50 \times 50$ $\times 15 \mathrm{~cm}$, length $\times$ width $\times$ height) for $5 \mathrm{~min}$ a day for 2 days to adapt to the environment. On the third day, two identical objects, A1 and A2 (made of wooden material), were placed in two adjacent corners of the arena approximately $10 \mathrm{~cm}$ from the edges. Mice were placed singly in the box and allowed to explore two objects for $5 \mathrm{~min}$. Exploration was defined as the mouse positioning its nose toward the object at a distance of less than $2 \mathrm{~cm}$ or touching the object. The mouse was returned to the home cage with 1-h inter-trial interval. The objects and the box were cleaned with ethanol (10\%) after each individual trial to avoid interference. Then, one object was replaced to a novel object B (made of the same material and color, only differing in shape). The mouse was returned to the box and allowed to explore the object for $5 \mathrm{~min}$ in the test phase. The exploration time (s) for each object in the test trials was recorded. A representation of the test is shown in Fig. 2.

The preferential index (PI) was calculated as [time spent exploring novel object/total exploration time].

\section{Morris water maze test (working memory)}

The Morris water maze (MWM) test was performed to measure spatial working memory ability. The water maze consisted of a circular black pool $(100 \mathrm{~cm}$ in diameter and $50 \mathrm{~cm}$ in height $)$ that was filled with water $\left(23 \pm 1{ }^{\circ} \mathrm{C}\right.$ and $30 \mathrm{~cm}$ in depth). A platform (10 $\mathrm{cm}$ in diameter) was placed $1 \mathrm{~cm}$ below the water surface. In the Morris water maze, we have the train phase before testing working memory. During the train phase, the mice were allowed to search for the submerged platform for no more than $60 \mathrm{~s}$ in quadrant IV for 5 days. Then, we started to test the working memory. The mice were trained five times for $60 \mathrm{~s}$ a day for three consecutive days to find the platform, which was placed in the pool at a different location (during the phase of testing working memory, day 1 , quadrant I; day 2 , quadrant II; day 3 , quadrant III). The escape latency (time required for each 

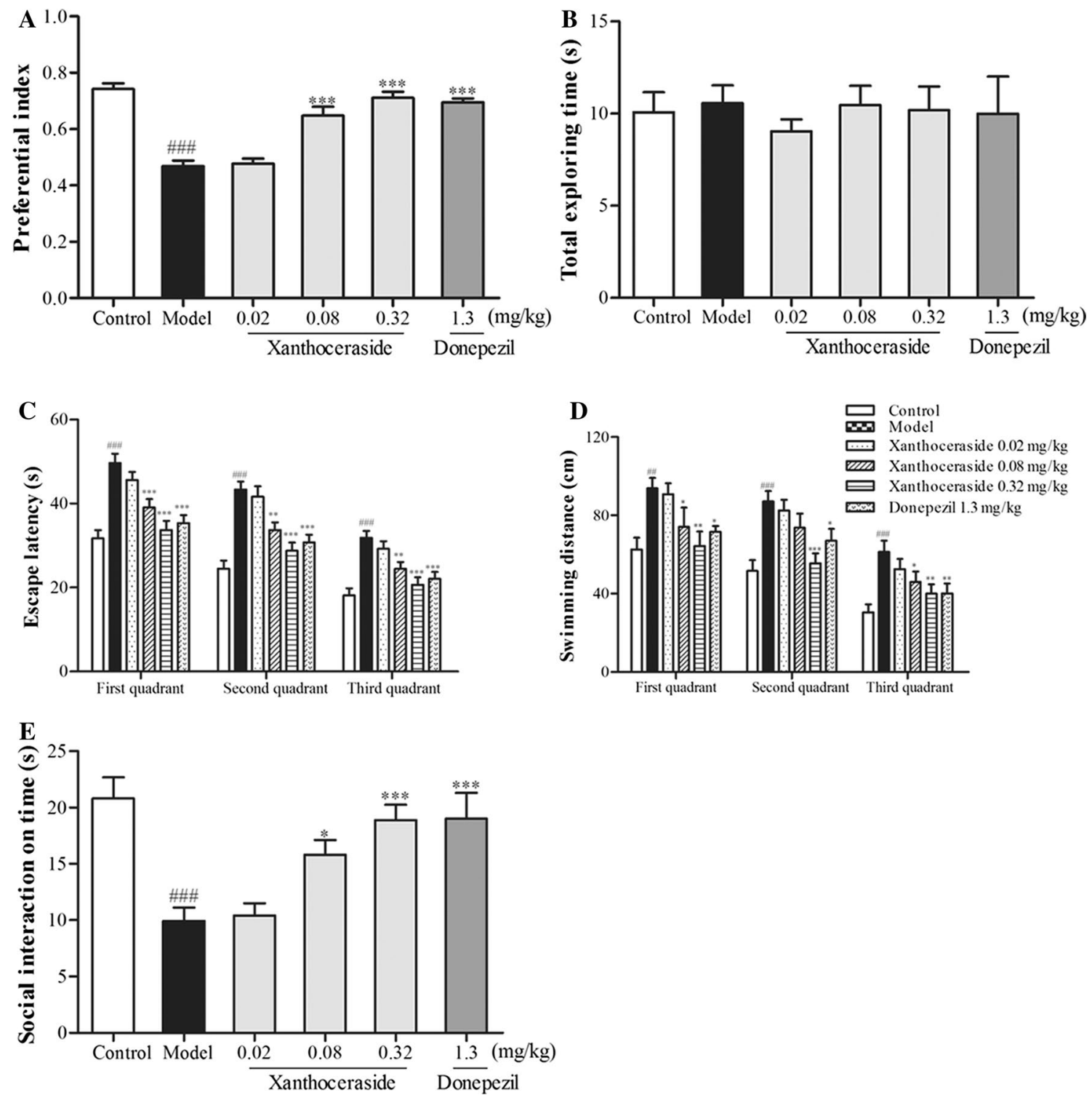

Fig. 2 Effects of xanthoceraside on learning and memory deficits and social behavior deficits in APP/PS1 transgenic mice. a Preferential index at $1 \mathrm{~h}$ in the retention session of the novel object recognition test. b Total exploration time $(1 \mathrm{~h})$ in the novel object recognition test. c, $\mathbf{d}$ Escape latency and swimming distance of working memory

mouse to find the platform) and swimming distance were recorded as an index of working memory.

\section{Social interaction test}

The social interaction test was conducted as we reported previously with minor modifications [33]. A mouse was housed alone in a home cage $(34 \times 22 \times 15 \mathrm{~cm})$ for 2 days before the test. Then, a novel mouse was placed into the cage together and allowed to explore freely for $5 \mathrm{~min}$ per trial. We recorded the time mice spent in social interactions, such as in the Morris water maze test. e Duration of interaction in the social interaction test. Values are expressed as the mean \pm SEM $(n=9-10)$. ${ }^{\# \#} p<0.01$ and ${ }^{\# \#} p<0.001$ versus the control group; $* p<0.05$, $* * p<0.01$, and $* * * p<0.001$ versus the model group

close following, inspection, face sniffing, anogenital sniffing, crawl-under/over behaviors and other social body contact.

\section{Transmission electron microscopy (TEM)}

Transmission electron microscopy was used to examine the ultrastructure of synapses in hippocampal slices [33]. Mice were anesthetized with $3 \%$ pentobarbital sodium $(30 \mathrm{mg} /$ $\mathrm{kg}$ i.p.) and fixed with $2.5 \%$ glutaraldehyde and $4 \%$ paraformaldehyde in $0.1 \mathrm{M}$ phosphate-buffered saline (temperature $4{ }^{\circ} \mathrm{C}, \mathrm{pH}$ 7.4). Hippocampal tissues of CA1 area were 
dissected as samples ( $n=2$ per group; two pictures for each tissue). The samples were divided into $1 \mathrm{~mm}^{3}$ pieces and post-fixed by incubation for $1 \mathrm{~h}$ in $2.5 \%$ glutaraldehyde at $4{ }^{\circ} \mathrm{C}$. Hippocampal ultrastructures, especially the synapse, were examined, and images were taken under a transmission electron microscope [Japan Electron Optical Laboratory (JEOL), Japan]. Synaptic impairment was identified by the decreasing presence of synaptic vesicles and the damage of synaptic structure.

\section{Golgi staining}

Golgi staining was performed as described previously [34] and the Golgi-Cox fixative solution included concentrations of mercury chloride, potassium chromate, potassium dichromate at $1 \%$. Briefly, the mice ( $n=4$ per group; 30 dendrites per group) were deeply anesthetized with $3 \%$ pentobarbital sodium (30 mg/kg i.p.), then transcardially perfused with $30 \mathrm{ml}$ of phosphate buffer saline. Brains were immersed in the fixative solution at $37^{\circ} \mathrm{C}$ for $24 \mathrm{~h}$ in darkness. The brain was sagittally sectioned using a vibrating microtome at a thickness of $200 \mu \mathrm{m}$ into PBS. The remained procedures were performed as described [35]. For measurement of spine density, only spines that emerged perpendicular to the dendritic shaft were counted in hippocampal CA1 region. We calculated the spine density as the number of spines per $10 \mu \mathrm{m}$.

\section{Western-blot analysis}

The protein concentrations were determined using a Bicinchoninic Acid Protein Assay Kit (CoWin Biosciences, China). The following procedure was described previously [36]. Briefly, the proteins ( $40 \mu \mathrm{g}$ of protein per lane) were run on $10 \%$ SDS gel and then electrophoretically transferred to polyvinyl difluoride (PVDF) membranes (Millipore, Bedford, MA, USA). After blocking with 5\% skim milk for $2 \mathrm{~h}$ at room temperature, the membranes were incubated with primary antibodies, rabbit anti-PSD95 (1:1000, Abcam, ab76115, USA), rabbit anti-SYP (1:1000, Abcam, ab32127, USA), rabbit anti-BDNF (1:1000, Abcam, ab108319, USA), rabbit anti-p-TrkB/anti-TrkB (1:500, Cell Signaling, 4621/4606, USA), rabbit anti-p-Akt/anti-Akt (1:500, Cell Signaling, 4060/4685, USA), rabbit anti-p-PI3K/anti-PI3K (1:800, Cell Signaling, 4228/4257, USA), mouse anti-pERK/anti-ERK (1:400, Santa Cruz, sc-81492/sc-514302, USA), rabbit anti-Rac1 (1:800, Cell Signaling, 2465, USA), rabbit anti-RhoA (1:1000, Cell Signaling, 2117, USA), rabbit anti-ROCK2 (1:800, Cell Signaling, 8236, USA), mouse anti-Snk (1:300, Santa Cruz, sc-374643, USA), goat antiSPAR (1:300, Santa Cruz, sc-31615, USA), rabbit antiCofilin (1:1000, Cell Signaling, 5175, USA) and mouse anti- $\beta$-actin (1:300, Santa Cruz, sc-47778, USA) at $4{ }^{\circ} \mathrm{C}$ overnight. The secondary antibodies including anti-mouse $\mathrm{IgG}$, anti-rabbit $\mathrm{IgG}$ and anti-goat $\operatorname{IgG}(1: 20,000$, Thermo Fisher, USA). Protein bands were visualized with an ECL Western blotting kit (CoWin Biosciences, China).

\section{Statistical analysis}

Statistical analysis was performed using SPSS 17.0 software. Data were expressed as the mean \pm SEM. Differences between the two groups were analyzed by Student's $t$ test. Statistical significance more than 2 groups were determined by one-way or two-way ANOVA followed by Fisher's least significant difference (LSD) multiple comparisons test. $P<0.05$ was considered to indicate statistical significance.

\section{Results}

\section{Effect of xanthoceraside on the impairments of imaginal memory, working memory, and social capability in APP/PS1 transgenic mice}

The effects of xanthoceraside on imaginal memory were evaluated using the novel object recognition test in APP/ PS1 transgenic mice. We observed that the level of exploratory preference for the novel objects (PI was calculated) in APP/PS1 transgenic mice was significantly decreased compared to the wild-type mice $\left(F_{5,54}=31.09, p<0.001\right.$, post hoc, $p<0.001$; Fig. 2a). Compared with the model group, xanthoceraside $(0.08$ and $0.32 \mathrm{mg} / \mathrm{kg})$ treated mice spent more time exploring the novel object $\mathrm{B}\left(F_{5,54}=31.09\right.$, $p<0.001$, post hoc, $p<0.001$; Fig. 2a). In addition, the total exploration time had no significant difference in each group $\left(F_{5,54}=0.139, p=0.983\right.$; Fig. $\left.2 b\right)$. These results indicated that xanthoceraside improved imaginal memory in APP/PS1 transgenic mice.

Next, we used the Morris water maze test to evaluate the effect of xanthoceraside on working memory in APP/PS1 transgenic mice. Compared with the control group, APP/ PS1 group mice spent longer time and distance to locate the platform (Fig. 2c, d). Compared with the APP/PS1 group, xanthoceraside treatment at $0.08 \mathrm{mg} / \mathrm{kg}$ and $0.32 \mathrm{mg} / \mathrm{kg}$ significantly decreased the escape latency (day $1: F_{5,52}=3.943$, $p<0.01$, day $2: F_{5,52}=5.746, p<0.001$, day $3: F_{5,52}=4.435$, $p<0.01$, post hoc, $p<0.05$; Fig. $2 \mathrm{c}$ ) and the swimming distance (day 1: $F_{5,54}=12.113, p<0.001$, day $2: F_{5,52}=13.393$, $p<0.001$, day $3: F_{5,52}=9.522, p<0.001$, post hoc, $p<0.05$; Fig. 2d) from day 1 to 3 . The effect of xanthoceraside was comparable to donepezil which was used as a positive control. These data showed that xanthoceraside prevented the impairments of spatial and working memory in APP/PS1 transgenic mice. 
In the social interaction test, compared with the control group, APP/PS1 group mice spent less time on active contacts with the peers. While, xanthoceraside at $0.08 \mathrm{mg} / \mathrm{kg}$ and $0.32 \mathrm{mg} / \mathrm{kg}$ significantly increased the duration of active contacts compared with the model group $\left(F_{5,54}=8.721\right.$, $p<0.001$, post hoc, $p<0.05$; Fig. 2e). Our results indicated that xanthoceraside treatment not only restored the memory deficits but also increased the ability of social interaction in APP/PS1 transgenic mice.

\section{Effect of xanthoceraside on synapse ultrastructure and the expression of SYP and PSD95 in the hippocampal of APP/PS1 transgenic mice}

Next, we used transmission electron microscopy to examine the ultrastructure of the hippocampus CA1 region. We found that the APP/PS1 group mice clearly showed the ambiguous synaptic structure (indicated by arrows) in the neurons of hippocampus (Fig. 3a). In contrast, the xanthoceraside treatment groups ameliorated the abnormal changes of synaptic structure in APP/PS1 transgenic mice (Fig. 3a). Next, we used Western-blot analysis to evaluate the effect of xanthoceraside on the expression of synaptic-associated proteins. Our results showed that the expression of SYP $\left(F_{4,15}=3.504, p<0.05\right.$, post hoc, $p<0.05$; Fig. $\left.3 b\right)$ and PSD95 $\left(F_{4,15}=3.107, p<0.05\right.$, post hoc, $p<0.05$; Fig. $\left.3 \mathrm{c}\right)$ were significantly reduced in the model group mice compared to the control group mice, indicating synaptic degeneration and reduction in the hippocampus of APP/PS1 transgenic mice. Xanthoceraside $(0.32 \mathrm{mg} / \mathrm{kg})$ significantly increased the expression of SYP and PSD95 (Fig. 3b, c).

\section{Effect of xanthoceraside on the expression of synapse-structure-associated signaling pathway in the hippocampal of APP/PS1 transgenic mice}

BDNF contributes to neuron and synapse development and the formation of hippocampus-dependent memory. Therefore, we investigated the effect of xanthoceraside on BDNF and its receptor TrkB in the hippocampus of APP/PS1
Fig. 3 Effects of xanthoceraside on synaptic deterioration and synapse-associated proteins in the hippocampus of APP/PS1 transgenic mice. a Representative transmission electron microscopic images of synaptic structures. A1: control group; B1: model group; C1: Xanthoceraside $0.02 \mathrm{mg} / \mathrm{kg}$ group; D1: Xanthoceraside $0.08 \mathrm{mg}$ / kg group; E1: Xanthoceraside $0.32 \mathrm{mg} / \mathrm{kg}$ group; F1: Donepezil $1.3 \mathrm{mg} / \mathrm{kg}$ group $(n=2$, $\times 40,000)$. b, c Western blots and quantification analysis of SYP and PSD95 expression in the hippocampus of APP/PS1 transgenic mice. Values are expressed as the mean \pm SEM $(n=4) .{ }^{\# \#} p<0.01$ versus the control group; $* p<0.05$ and $* * p<0.01$ versus the model group
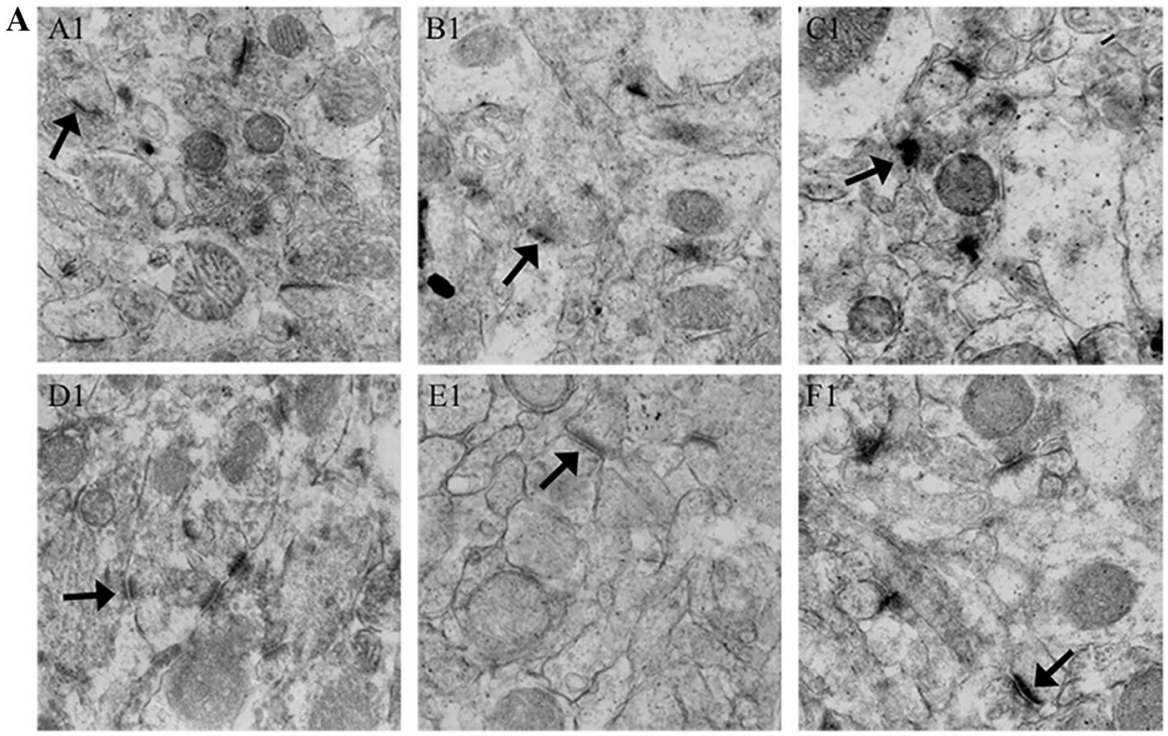

B
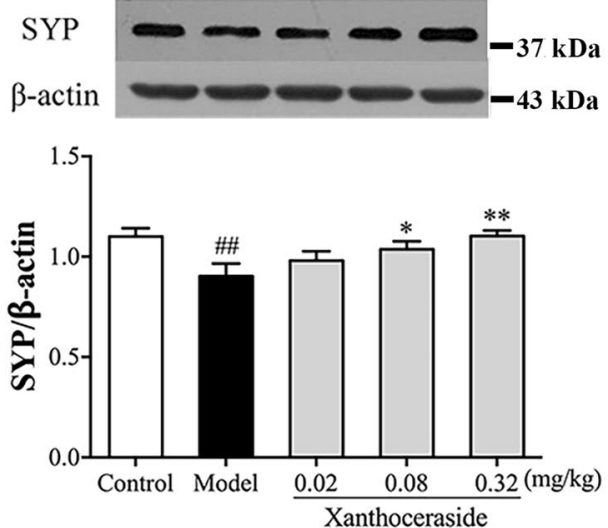

C
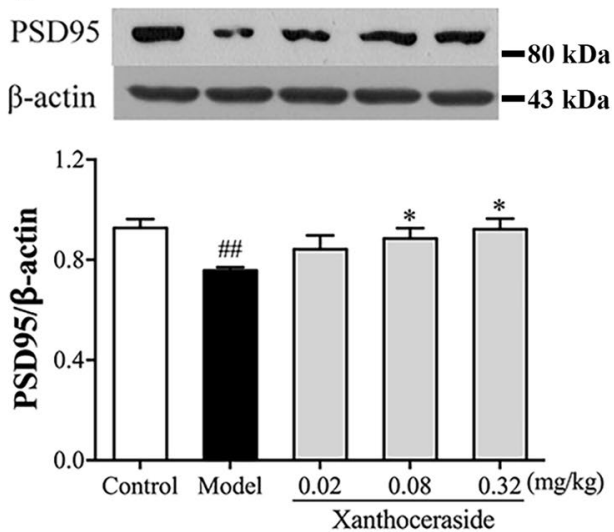
Fig. 4 Effects of xanthoceraside on the expression of synapse-structure-associated signaling pathway proteins in the hippocampus of APP/ PS1 transgenic mice. a-e Representative Western blots and quantification analysis of BDNF, TrkB, p-ERK, p-PI3K and p-Akt in the hippocampus of APP/PS1 transgenic mice. Values are expressed as the mean $\pm \operatorname{SEM}(n=4) .{ }^{\# \#} p<0.01$ and ${ }^{\# \# \#} p<0.001$ versus the control group; $* p<0.05, * * p<0.01$ and $* * * p<0.001$ versus the model group
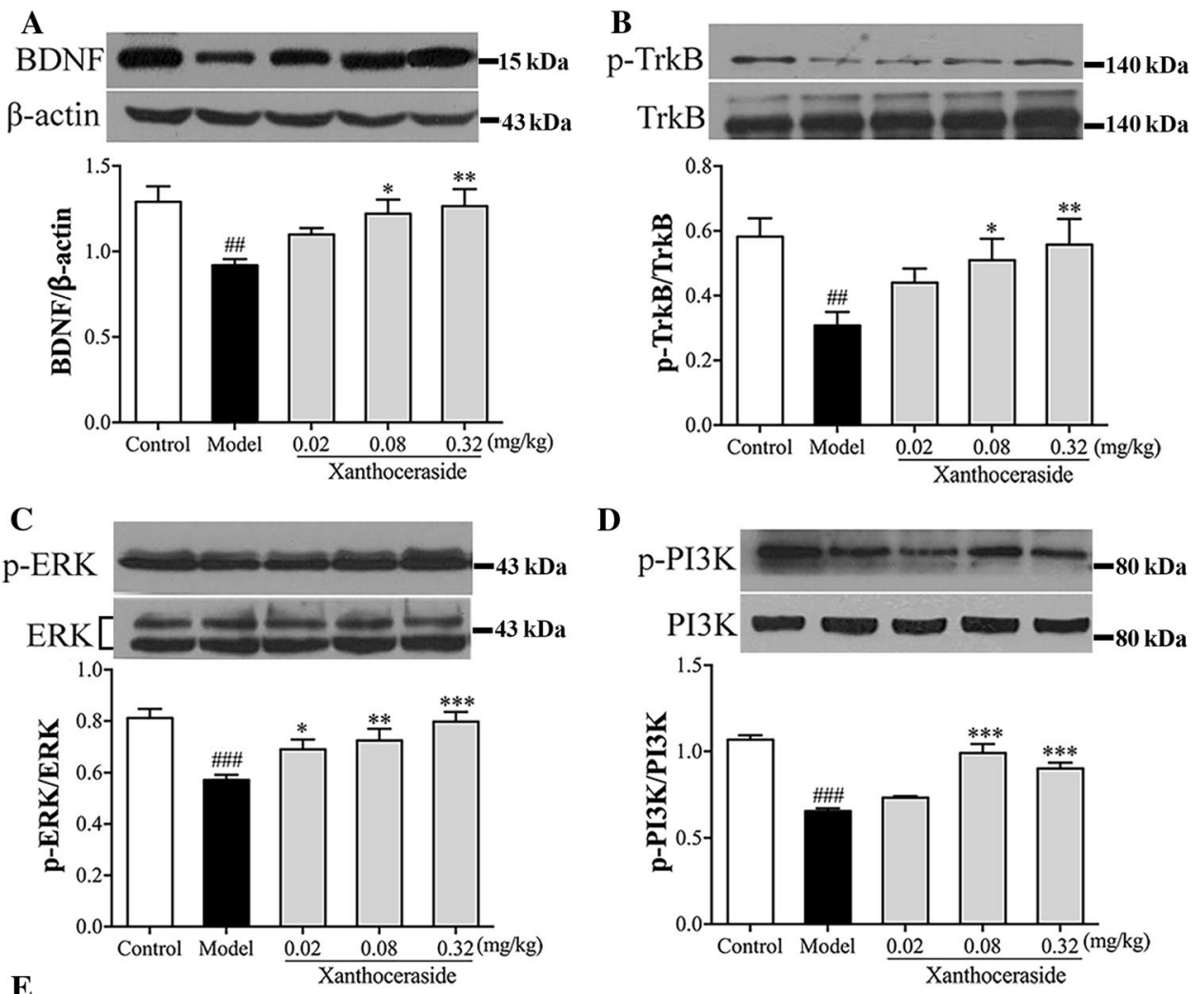

D
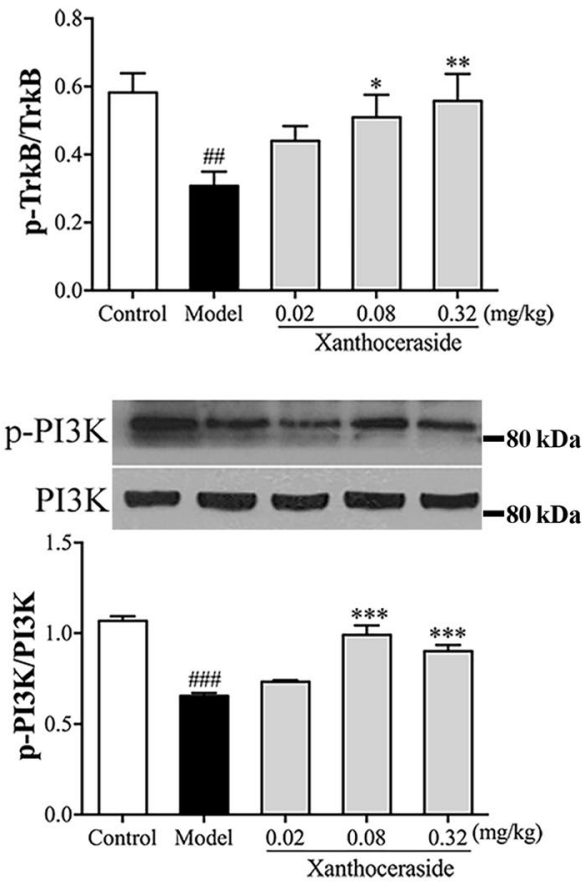

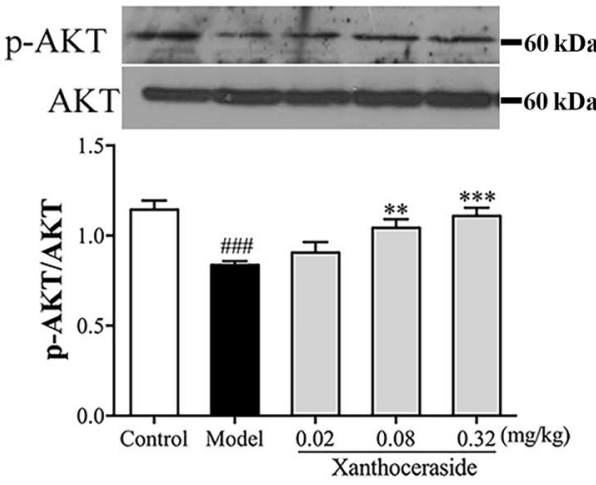

transgenic mice. Western-blot analysis showed that, compared with the control group mice, the expression of BDNF $\left(F_{4,15}=4.263, p<0.05\right.$, post hoc, $p<0.05$; Fig. $\left.4 \mathrm{a}\right)$ and the phosphorylation level of $\operatorname{TrkB}\left(F_{4,15}=3.517, p<0.05\right.$, post hoc, $p<0.05$; Fig. $4 \mathrm{~b})$ were significantly decreased in the model group. Xanthoceraside $(0.08$ and $0.32 \mathrm{mg} / \mathrm{kg}) \mathrm{sig}-$ nificantly increased the expression of BDNF and the levels of phosphorylated TrkB (Fig. 4a, b).

MAPK/ERK and PI3K/Akt are two major downstream targets of BNDF. Therefore, we investigated the effect of xanthoceraside on MAPK/ERK and PI3K/Akt signaling pathways in the hippocampus of APP/PS1 transgenic mice. Western-blot analysis showed that, compared with the control group mice, the phosphorylation levels of p-ERK $\left(F_{4,15}=7.176, p<0.01\right.$, post hoc, $p<0.05$; Fig. $\left.4 \mathrm{c}\right)$, p-PI3K $\left(F_{4,15}=28.963, p<0.001\right.$, post hoc, $p<0.001$; Fig. $\left.4 d\right)$ and
p-Akt $\left(F_{4,15}=8.258, p<0.001\right.$, post hoc, $p<0.01$; Fig. $\left.4 \mathrm{e}\right)$ were significantly decreased in the model group. Xanthoceraside $(0.08 \mathrm{mg} / \mathrm{kg}$ and $0.32 \mathrm{mg} / \mathrm{kg})$ significantly increased the expression of phosphorylated ERK, PI3K, and Akt (Fig. 4c-e).

\section{Effect of xanthoceraside on dendritic spine density and associated protein expression in the hippocampus of APP/PS1 transgenic mice}

Dendritic spine is important to maintain synaptic plasticity. Next, we assessed the effect of xanthoceraside on dendritic spine density using the Golgi staining (Fig. 5a). Our results showed that dendritic spine density was decreased in the model group $\left(F_{3,116}=11.844, p<0.001\right.$, post hoc, $p<0.01$; Fig. 5b). Xanthoceraside increased the dendritic 
Fig. 5 Effects of xanthoceraside on dendritic spine density and expression of RhoA, ROCK2, Snk, and SPAR proteins in the hippocampus of APP/PS1 transgenic mice. a Representative pictures of Golgi staining (×1000). b Quantification analysis of dendritic spine density $(n=4,30$ dendrites per group). c-f Western blots and quantification analysis of RhoA, ROCK2, Snk, and SPAR in APP/PS1 transgenic mice. Values are expressed as the mean $\pm \operatorname{SEM}(n=4) .{ }^{\#} p<0.05$, ${ }^{\# \#} p<0.01$, and ${ }^{\# \# \# p} p<0.001$ versus the control group, $* p<0.05$, $* * p<0.01$, and $* * * p<0.001$ versus the model group
A

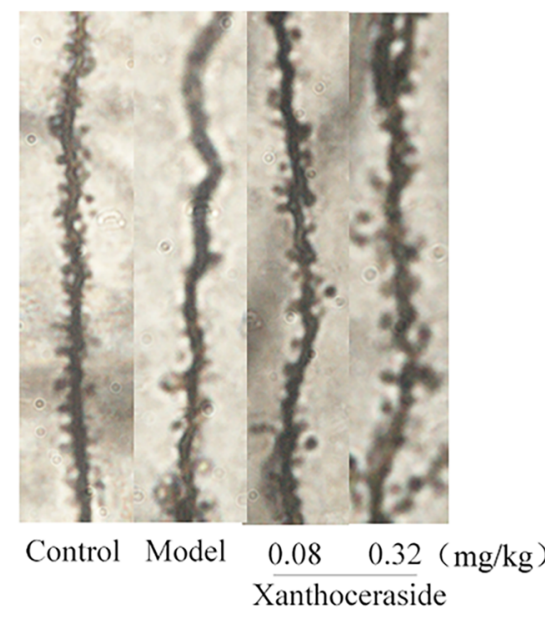

C
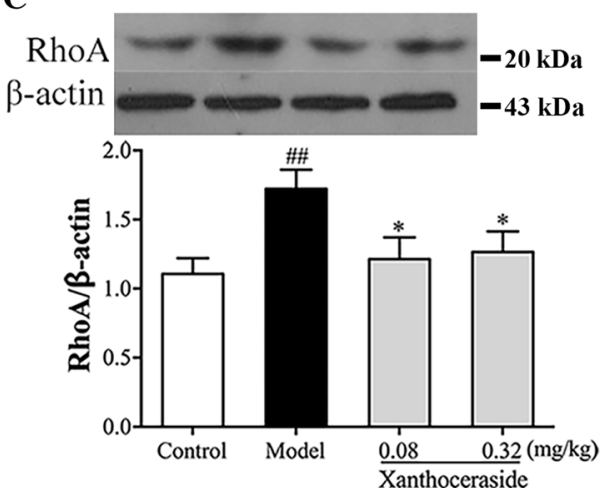

E
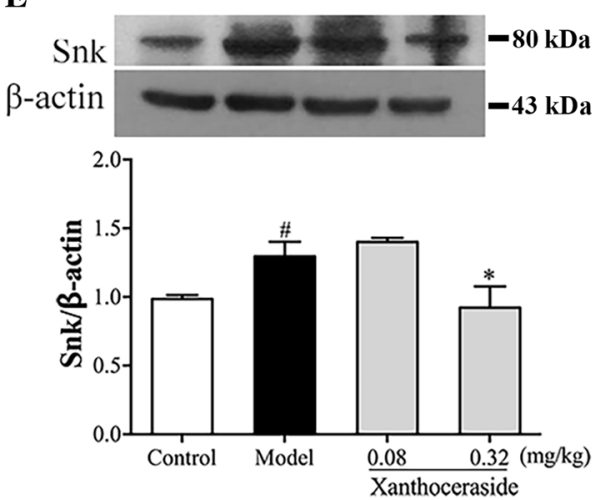

B

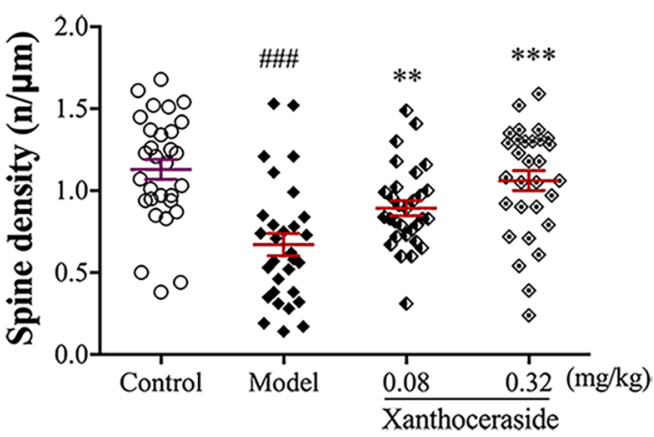

D
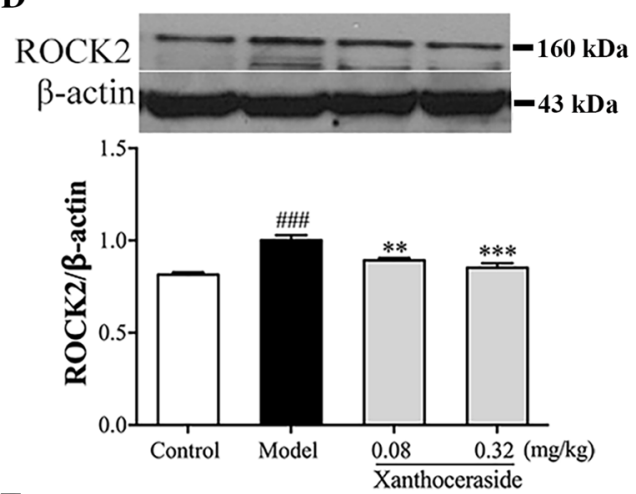

F
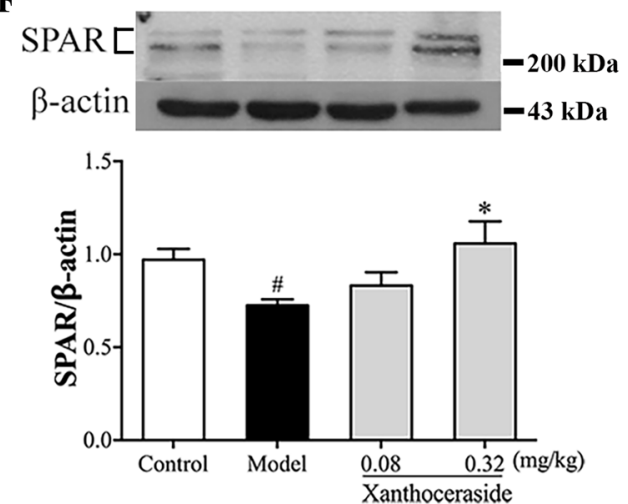

spine density significantly compared to the model group in the hippocampus of APP/PS 1 transgenic mice $\left(F_{3,116}=11.844, p<0.001\right.$, post hoc, $p<0.01$; Fig. $\left.5 b\right)$. We next investigated the expression of proteins are associated with dendritic spine formation. We observed that treatment with xanthoceraside significantly decreased the expression of RhoA $\left(F_{3,12}=3.762, p<0.05\right.$, post hoc, $p<0.05$; Fig. 5c), ROCK2 $\left(F_{3,12}=17.047, p<0.001\right.$, post hoc, $p<0.01$; Fig. 5d) and Snk $\left(F_{3,12}=5.900, p<0.05\right.$, post hoc, $p<0.05$; Fig. 5e), but increased $\operatorname{SPAR}\left(F_{3,12}=3.622\right.$, $p<0.05$, post hoc, $p<0.05$; Fig. 5f) in APP/PS1 transgenic mice.

\section{Effect of xanthoceraside on BDNF/TrkB/ cofilin-associated signaling pathway proteins in the hippocampus of APP/PS1 transgenic mice}

In our study, we had identified that the expression of BDNF and the phosphorylation of TrkB were significantly increased in APP/PS1 transgenic mice treatment with xanthoceraside (Fig. 4a, b). BDNF/TrkB, as well as the downstream proteins of Rac1 and cofilin, played an important role in regulating the stability of synapses [25]. Next, we detected the proteins expression of Rac1 and cofilin. The results showed that Rac1 was significantly decreased $\left(F_{3,12}=4.934, p<0.05\right.$, post hoc, 
Fig. 6 Effects of xanthoceraside on the expression of BDNF/

TrkB/cofilin in the hippocampus of APP/PS1 transgenic mice. a, b Representative Western blots and quantification analysis of Rac1 and cofilin in APP/PS1 transgenic mice. Values are expressed as the mean \pm SEM $(n=4) .{ }^{\# \#} p<0.01$ and

\#\#\# $p<0.001$ versus the control group, $* p<0.05$ and $* * p<0.01$ versus the model group

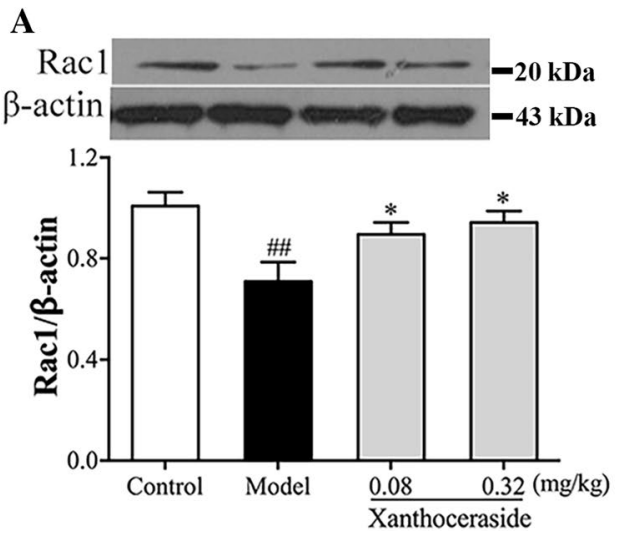

$p<0.05$; Fig. 6a), while cofilin was increased $\left(F_{3,12}=7.591\right.$, $p<0.01$, post hoc, $p<0.05$; Fig. $6 \mathrm{~b}$ ) in APP/PS1 transgenic mice. However, xanthoceraside treatment could significantly reverse the abnormal expression of these proteins in APP/ PS1 transgenic mice (Fig. 6a, b).

\section{Discussion}

Our previous study reported that xanthoceraside treatment significantly improved cognitive impairments, prevented the loss of cholinergic neurons, inhibited tau hyper-phosphorylation and NF- $\mathrm{KB}$ signaling pathway, as well as activated MAPK and Ras-ERK signaling pathways in several AD animal models [29, 32, 37]. In the clinic, the symptoms of $\mathrm{AD}$ patients are not only cognitive impairment but also anxiety and obstacles to social activities [38-40]. Herein, we firstly found that xanthoceraside treatment also attenuated the spatial memory impairment in 10-month-old APP/ PS1 transgenic mice in the Morris water maze test. Previous study shows the protective effect of xanthoceraside on big difference of novel object in novel recognition test [41, 42]. In this study, we detected the small different object in novel object recognition test, further to discuss the protective role in imaginal memory. Results found that treatment with xanthoceraside significantly improved the imaginal memory impairment in novel object recognition test. Meanwhile, we provided the first evidence that xanthoceraside treatment attenuated the deficits of social activities in the social interaction test. Donepezil, one of the acetylcholinesterase inhibitors, is used for the treatment of AD patients [43] and usually used as a positive drug for basic research [44]. Xanthoceraside $(0.32 \mathrm{mg} / \mathrm{kg})$ had better results in behavioral tests than donepezil $(1.3 \mathrm{mg} / \mathrm{kg})$.

Synapse damage and loss strongly correlate with cognitive decline in the progress of AD [45]. Down-regulation of synaptophysin (SYP) and post-synaptic density protein (PSD95) in the brains of AD animal models contribute to synaptic dysfunction and behavioral impairment [46].
Synaptic vesicle associated protein SYP has been considered the marker of synaptic plasticity in neural networks [47]. To some extent, the decreased expression of synaptic vesicle-associated protein SYP reflects synaptic impairment. Some literature has reported that $A \beta$ is toxic to synapses [48]. In AD patients and animal models, there is a significant reduction in both the dendritic spine density and the number of SYP-positive synapses near senile plaques $[49,50]$. In our study, we found that synaptic structure was ambiguous; the expression of major synaptic markers SYP and PSD95 were significantly reduced in the hippocampus of APP/PS1 transgenic mice, but were ameliorated after xanthoceraside treatment. Our previous research indicated that xanthoceraside decreased the aggregation of $A \beta$ by reducing the production of APP [41]. This paper further suggested that the anti-synaptotoxicitic effects of xanthoceraside also might be one of the reasons to ameliorate learning and memory impairments in $\mathrm{AD}$ animal models.

BDNF is one of the important factors in maintaining the survival and regeneration of synapses, and it is also required for neuronal survival and appropriated function of hippocampus, parietal cortex, basal forebrain, and some other areas associated with AD [51]. BDNF and the phosphorylation of its receptor, TrkB, as one of the factors in activating several signaling pathways, such as MAPK/ERK, PI3K/Akt, and Rac1/cofilin ameliorate synaptic impairment $[25,52]$. PI3K/Akt plays a central role in neuronal protein synthesis, neuronal survival, and synaptic plasticity, through it inhibits the expression of pro-apoptotic proteins and regulates the function of some transcription factors [53]. Activation of PI3K/Akt triggers many downstream targets, including GSK-3 $\beta$, mTOR, p70S6K1, Bad, eNOS, and Forkhead, which are implicated in cognitive decline, neuronal cell death, oxidative stress, angiogenesis, and tau hyperphosphorylation [54-56]. Studies reported that A $\beta$ inhibited the BDNF/TrkB pathway and TrkB phosphorylation [34], and reduced the expression of its downstream target MAPK/ERK and PI3K/Akt in brain of APP/PS1 transgenic mice [57]. In our study, we mainly investigated the effect 
of xanthoceraside on BDNF/TrkB, as well as its downstream pathways. A study showed that the level of BDNF was significantly decreased in the hippocampus of APP/PS1 transgenic mice but attenuated by xanthoceraside treatment. Xanthoceraside also alleviated the inhibition of TrkB phosphorylation and increased phosphorylated ERK expression in APP/PS1 transgenic mice. Moreover, the down-regulation of phosphorylated PI3K/Akt was significantly attenuated by xanthoceraside treatment. These results suggested that xanthoceraside protected the function of neurons and synapses through the BDNF/TrkB/ERK and PI3K/Akt cascade.

Dendritic regression occurs in several brain regions of AD patients, which has been found to be associated with the cognitive dysfunction of AD patients and model mice [58, 59]. To further investigate the protective effects of xanthoceraside on synaptic structural abnormalities, we detected the morphology change of dendritic spines. Our data showed that dendritic spine density was decreased in AD mice, while treatment with xanthoceraside significantly increased the density of dendritic spine. Other reports showed that RhoA increased in AD mice and played an important role in AD pathogenesis via ROCK activation, which induced the limitation of tubulin assembly in neuritis, and disorders of the neuronal morphology, thus led to cognitive impairment [49, 60]. In our study, we also found that expressions of RhoA and ROCK were increased in AD mice and xanthoceraside significantly reversed these abnormal expressions. SPAR, a large multimodular scaffold protein in the PSD, combines with a complex of PSD-95 to regulate spine morphogenesis [61]. Snk induces the degradation of SPAR, further leading to spine shrinkage and loss. Our results showed that xanthoceraside significantly inhibited Snk expression and increased SPAR to protect the dendritic spine loss in APP/PS1 transgenic mice. Cofilin is an important member of the actin depolymerization factors, which cleaves and depolymerizes fibroactin, causing dendritic spines to atrophy and degenerate. The decreased BDNF/TrkB protein expression could reduce the expression of Rac1 [25], and lead to the abnormal increase of cofilin in AD mice [62]. Consistent with the papers, we found that the expression of BDNF, $\mathrm{p}-\mathrm{TrkB}$, and Rac1 was decreased, while cofilin was increased in APP/PS1 transgenic mice. Xanthoceraside significantly increased the expression of BDNF, p-TrkB, and Rac1, and inhibited the expression of cofilin in APP/PS1 transgenic mice.

In conclusion, our results indicated the potential benefits of xanthoceraside against cognitive deficits and synapse disorders in APP/PS1 transgenic mice. The possible mechanism could be related to the regulation of the BDNF/TrkB signaling pathway and the enriched dendritic spine density.

Authors' contribution G. Jin and L. Zhu conceived the experiments and contributed to research data; P. Liu, Q. Xu and Y.Q. contributed technical assistance; X. Zou and J. Xu participated in raising animals; and X. Ji and T. Chi revised the manuscript. L. Zou revised the manuscript and supervised the analyses.

Funding This work was supported by the Research Fund for National Natural Science Foundation (No. 81373992) of China, the Career Development Program for Young Teachers in Shenyang Pharmaceutical University (No. ZQN2015028) and the Doctoral Scientific Research Foundation of Liaoning Province (No. 51120424).

\section{Compliance with ethical standards}

Conflict of interest The authors have no conflicts of interest to declare.

Ethical approval Animal studies were performed in accordance with the P.R. China legislation on the use and the care of laboratory animals and with the guidelines established by the Institute for Experimental Animals at Shenyang Pharmaceutical University. The protocols were approved by the Committee on the Ethics of Animal Experiments of the Shenyang Pharmaceutical University (SYPU-IACUC-S20140317-04).

\section{References}

1. Dong H, Csernansky CA, Martin MV, Bertchume A, Vallera D, Csernansky JG (2005) Acetylcholinesterase inhibitors ameliorate behavioral deficits in the $\mathrm{Tg} 2576$ mouse model of Alzheimer's disease. Psychopharmacology 181(1):145-152

2. Cras P, Kawai M, Lowery D, Gonzalez-DeWhitt P, Greenberg B, Perry G (1991) Senile plaque neurites in Alzheimer disease accumulate amyloid precursor protein. Proc Natl Acad Sci USA 88(17):7552-7556

3. Kawai M, Cras P, Richey P, Tabaton M, Lowery DE, GonzalezDeWhitt PA, Greenberg BD, Gambetti P, Perry G (1992) Subcellular localization of amyloid precursor protein in senile plaques of Alzheimer's disease. Am J Pathol 140(4):947-958

4. Grundke-Iqbal I, Iqbal K, Tung YC, Quinlan M, Wisniewski HM, Binder LI (1986) Abnormal phosphorylation of the microtubuleassociated protein tau (tau) in Alzheimer cytoskeletal pathology. Proc Natl Acad Sci USA 83(13):4913-4917

5. Garcia P, Youssef I, Utvik JK, Florent-Bechard S, Barthelemy V, Malaplate-Armand C, Kriem B, Stenger C, Koziel V, Olivier JL et al (2010) Ciliary neurotrophic factor cell-based delivery prevents synaptic impairment and improves memory in mouse models of Alzheimer's disease. J Neurosci 30(22):7516-7527

6. Price KA, Varghese M, Sowa A, Yuk F, Brautigam H, Ehrlich ME, Dickstein DL (2014) Altered synaptic structure in the hippocampus in a mouse model of Alzheimer's disease with soluble amyloid-beta oligomers and no plaque pathology. Mol Neurodegener 9:41

7. Raskin J, Cummings J, Hardy J, Schuh K, Dean RA (2015) Neurobiology of Alzheimer's disease: integrated molecular, physiological, anatomical, biomarker, and cognitive dimensions. Curr Alzheimer Res 12(8):712-722

8. Calkins MJ, Manczak M, Mao P, Shirendeb U, Reddy PH (2011) Impaired mitochondrial biogenesis, defective axonal transport of mitochondria, abnormal mitochondrial dynamics and synaptic degeneration in a mouse model of Alzheimer's disease. Hum Mol Genet 20(23):4515-4529

9. Strittmatter S, Kostylev M, Kaufman AC, Haas L, Heiss JK, Gunther EC, Nygaard HB (2014) A synaptotoxic pathway from $\mathrm{A} \beta$ oligomer to prion protein to mGluR5 to F kinase in Alzheimer's disease. Neurobiol Aging 35:S21 
10. Chapman PF, White GL, Jones MW, Cooper-Blacketer D, Marshall VJ, Irizarry M, Younkin L, Good MA, Bliss TV, Hyman BT et al (1999) Impaired synaptic plasticity and learning in aged amyloid precursor protein transgenic mice. Nat Neurosci 2(3):271-276

11. Pooler AM, Noble W, Hanger DP (2014) A role for tau at the synapse in Alzheimer's disease pathogenesis. Neuropharmacology 76(Pt A): $1-8$

12. Gong B, Vitolo OV, Trinchese F, Liu S, Shelanski M, Arancio O (2004) Persistent improvement in synaptic and cognitive functions in an Alzheimer mouse model after rolipram treatment. J Clin Invest 114(11):1624-1634

13. Bonardi C, Pardon MC, Armstrong P (2016) Deficits in objectin-place but not relative recency performance in the APPswe/ PS1dE9 mouse model of Alzheimer's disease: implications for object recognition. Behav Brain Res 313:71-81

14. Li N, Liu GT (2010) The novel squamosamide derivative FLZ enhances BDNF/TrkB/CREB signaling and inhibits neuronal apoptosis in APP/PS1 mice. Acta Pharmacol Sin 31(3):265-272

15. Hassanpour M, Rezabakhsh A, Rahbarghazi R, Nourazarian A, Nouri M, Avci CB, Ghaderi S, Alidadyani N, Bagca BG, Bagheri HS (2017) Functional convergence of Akt protein with VEGFR-1 in human endothelial progenitor cells exposed to sera from patient with type 2 diabetes mellitus. Microvasc Res 114:101-113

16. Wang X, Fu X, Zhao S, Zhang H, Shao L, Li G, Fan C (2017) Antiangiogenic properties of caudatin in vitro and in vivo by suppression of VEGFVEGFR2AKT/FAK signal axis. Mol Med Rep 16(6):8937-8943

17. Wang WM, Xu Y, Wang YH, Sun HX, Sun YF, He YF, Zhu QF, Hu B, Zhang X, Xia JL et al (2017) HOXB7 promotes tumor progression via bFGF-induced activation of MAPK/ERK pathway and indicated poor prognosis in hepatocellular carcinoma. Oncotarget 8(29):47121-47135

18. Xiong S, Xu Y, Ma M, Wang H, Wei F, Gu Q, Xu X (2017) Neuroprotective effects of a novel peptide, FK18, under oxygen-glucose deprivation in SH-SY5Y cells and retinal ischemia in rats via the Akt pathway. Neurochem Int 108:78-90

19. Je HS, Yang F, Ji Y, Nagappan G, Hempstead BL, Lu B (2012) Role of pro-brain-derived neurotrophic factor (proBDNF) to mature BDNF conversion in activity-dependent competition at developing neuromuscular synapses. Proc Natl Acad Sci USA 109(39):15924-15929

20. Tapia-Arancibia L, Aliaga E, Silhol M, Arancibia S (2008) New insights into brain BDNF function in normal aging and Alzheimer disease. Brain Res Rev 59(1):201-220

21. Khelfaoui M, Denis C, van Galen E, de Bock F, Schmitt A, Houbron C, Morice E, Giros B, Ramakers G, Fagni L et al (2007) Loss of X-linked mental retardation gene oligophrenin1 in mice impairs spatial memory and leads to ventricular enlargement and dendritic spine immaturity. J Neurosci 27(35):9439-9450

22. Schmandke A, Strittmatter SM (2007) ROCK and Rho: biochemistry and neuronal functions of Rho-associated protein kinases. Neuroscientist 13(5):454-469

23. Wu LX, Sun CK, Zhang YM, Fan M, Xu J, Ma H, Zhang J (2007) Involvement of the Snk-SPAR pathway in glutamate-induced excitotoxicity in cultured hippocampal neurons. Brain Res 1168:38-45

24. Bennett M (2009) Positive and negative symptoms in schizophrenia: the NMDA receptor hypofunction hypothesis, neuregulin/ErbB4 and synapse regression. Aust N Z J Psychiatry 43(8):711-721

25. Bennett MR, Lagopoulos J (2014) Stress and trauma: BDNF control of dendritic-spine formation and regression. Prog Neurobiol 112:80-99

26. Lu B, Nagappan G, Guan X, Nathan PJ, Wren P (2013) BDNFbased synaptic repair as a disease-modifying strategy for neurodegenerative diseases. Nat Rev Neurosci 14(6):401-416
27. Peng S, Wuu J, Mufson EJ, Fahnestock M (2005) Precursor form of brain-derived neurotrophic factor and mature brain-derived neurotrophic factor are decreased in the pre-clinical stages of Alzheimer's disease. J Neurochem 93(6):1412-1421

28. Arancibia S, Silhol M, Mouliere F, Meffre J, Hollinger I, Maurice T, Tapia-Arancibia L (2008) Protective effect of BDNF against beta-amyloid induced neurotoxicity in vitro and in vivo in rats. Neurobiol Dis 31(3):316-326

29. Chi TY, Wang LH, Qu C, Yang BZ, Ji XF, Wang Y, Okuyama T, Yoshihito O, Zou LB (2009) Protective effects of xanthoceraside on learning and memory impairment induced by Abeta(25-35) in mice. J Asian Nat Prod Res 11(12):1019-1027

30. Lu P, Mamiya T, Lu L, Mouri A, Ikejima T, Kim HC, Zou LB, Nabeshima T (2012) Xanthoceraside attenuates amyloid beta peptide $(2)(5)(-)(3)(5)$-induced learning and memory impairments in mice. Psychopharmacology 219(1):181-190

31. Liu P, Zou LB, Wang LH, Jiao Q, Chi TY, Ji XF, Jin G (2014) Xanthoceraside attenuates tau hyperphosphorylation and cognitive deficits in intracerebroventricular-streptozotocin injected rats. Psychopharmacology 231(2):345-356

32. Qi Y, Zou LB, Wang LH, Jin G, Pan JJ, Chi TY, Ji XF (2013) Xanthoceraside inhibits pro-inflammatory cytokine expression in Abeta25-35/IFN-gamma-stimulated microglia through the TLR2 receptor, MyD88, nuclear factor-kappaB, and mitogenactivated protein kinase signaling pathways. J Pharmacol Sci 122(4):305-317

33. Zhou L, Gao Q, Nie M, Gu JL, Hao W, Wang L, Cao JM (2016) Degeneration and energy shortage in the suprachiasmatic nucleus underlies the circadian rhythm disturbance in ApoE-/- mice: implications for Alzheimer's disease. Sci Rep 6:36335

34. Li Y, Xu J, Xu P, Song S, Liu P, Chi T, Ji X, Jin G, Qiu S, Hou Y et al (2016) Xanthoceras sorbifolia extracts ameliorate dendritic spine deficiency and cognitive decline via upregulation of BDNF expression in a rat model of Alzheimer's disease. Neurosci Lett 629:208-214

35. Baloyannis SJ (2015) Staining neurons with Golgi techniques in degenerative diseases of the brain. Neural Regen Res 10(5):693-695

36. Zhu L, Chi T, Zhao X, Yang L, Song S, Lu Q, Ji X, Liu P, Wang L, Zou L (2018) Xanthoceraside modulates neurogenesis to ameliorate cognitive impairment in APP/PS1 transgenic mice. J Physiol Sci 68(5):555-565

37. Liu P, Zou L, Jiao Q, Chi T, Ji X, Qi Y, Xu Q, Wang L (2013) Xanthoceraside attenuates learning and memory deficits via improving insulin signaling in STZ-induced AD rats. Neurosci Lett 543:115-120

38. Pietropaolo S, Delage P, Lebreton F, Crusio WE, Cho YH (2012) Early development of social deficits in APP and APP-PS1 mice. Neurobiol Aging 33(5):1002 e1017-1002 e1027

39. Nenov MN, Laezza F, Haidacher SJ, Zhao Y, Sadygov RG, Starkey JM, Spratt H, Luxon BA, Dineley KT, Denner L (2014) Cognitive enhancing treatment with a PPARgamma agonist normalizes dentate granule cell presynaptic function in Tg2576 APP mice. J Neurosci 34(3):1028-1036

40. Liang C, Tan S, Huang Q, Lin J, Lu Z, Lin X (2015) Pratensein ameliorates beta-amyloid-induced cognitive impairment in rats via reducing oxidative damage and restoring synapse and BDNF levels. Neurosci Lett 592:48-53

41. Jin G, Wang LH, Ji XF, Chi TY, Qi Y, Jiao Q, Xu Q, Zhou XY, Zhang R, Zou LB (2014) Xanthoceraside rescues learning and memory deficits through attenuating beta-amyloid deposition and tau hyperphosphorylation in APP mice. Neurosci Lett 573:58-63

42. Zhu L, Yang L, Zhao X, Liu D, Guo X, Liu P, Chi T, Ji X, Zou L (2018) Xanthoceraside modulates NR2B-containing NMDA receptors at synapses and rescues learning-memory deficits in APP/PS1 transgenic mice. Psychopharmacology 235(1):337-349 
43. Kaushik V, Smith ST, Mikobi E, Raji MA (2018) Acetylcholinesterase inhibitors: beneficial effects on comorbidities in patients with Alzheimer's disease. Am J Alzheimers Dis Other Demen 33(2):73-85

44. Ding N, Jiang J, Lu M, Hu J, Xu Y, Liu X, Li Z (2017) Manual acupuncture suppresses the expression of proinflammatory proteins associated with the NLRP3 inflammasome in the hippocampus of SAMP8 mice. Evid Based Complement Alternat Med 2017:3435891

45. Counts SE, Alldred MJ, Che S, Ginsberg SD, Mufson EJ (2014) Synaptic gene dysregulation within hippocampal CA1 pyramidal neurons in mild cognitive impairment. Neuropharmacology 79:172-179

46. Chai JX, Li HH, Wang YY, Chai Q, He WX, Zhou YM, Hu XD, Wang ZH (2016) Effect of diallyl disulfide on learning and memory abilities and hippocampal synapses in mouse models of Alzheimer's disease. Nan Fang Yi Ke Da Xue Xue Bao 36(10):1417-1422

47. Smith TD, Adams MM, Gallagher M, Morrison JH, Rapp PR (2000) Circuit-specific alterations in hippocampal synaptophysin immunoreactivity predict spatial learning impairment in aged rats. J Neurosci 20(17):6587-6593

48. Hoppe JB, Haag M, Whalley BJ, Salbego CG, Cimarosti H (2013) Curcumin protects organotypic hippocampal slice cultures from Abeta1-42-induced synaptic toxicity. Toxicol In Vitro 27(8):2325-2330

49. Tsai J, Grutzendler J, Duff K, Gan WB (2004) Fibrillar amyloid deposition leads to local synaptic abnormalities and breakage of neuronal branches. Nat Neurosci 7(11):1181-1183

50. Dong H, Martin MV, Chambers S, Csernansky JG (2007) Spatial relationship between synapse loss and beta-amyloid deposition in Tg2576 mice. J Comp Neurol 500(2):311-321

51. Tucsek Z, Noa Valcarcel-Ares M, Tarantini S, Yabluchanskiy A, Fulop G, Gautam T, Orock A, Csiszar A, Deak F, Ungvari Z (2017) Hypertension-induced synapse loss and impairment in synaptic plasticity in the mouse hippocampus mimics the aging phenotype: implications for the pathogenesis of vascular cognitive impairment. Geroscience 39:385-406

52. Bruel-Jungerman E, Veyrac A, Dufour F, Horwood J, Laroche S, Davis S (2009) Inhibition of PI3K-Akt signaling blocks exercisemediated enhancement of adult neurogenesis and synaptic plasticity in the dentate gyrus. PLoS ONE 4(11):e7901

53. Morroni F, Sita G, Tarozzi A, Rimondini R, Hrelia P (2016) Early effects of Abeta1-42 oligomers injection in mice: involvement of
PI3K/Akt/GSK3 and MAPK/ERK1/2 pathways. Behav Brain Res 314:106-115

54. Uranga RM, Katz S, Salvador GA (2013) Enhanced phosphatidylinositol 3-kinase (PI3K)/Akt signaling has pleiotropic targets in hippocampal neurons exposed to iron-induced oxidative stress. J Biol Chem 288(27):19773-19784

55. On C (2013) PI3-kinase/Akt/mTOR signaling: impaired on/off switches in aging, cognitive decline and Alzheimer's disease. Exp Gerontol 48(7):647-653

56. Hoppe JB, Coradini K, Frozza RL, Oliveira CM, Meneghetti AB, Bernardi A, Pires ES, Beck RC, Salbego CG (2013) Free and nanoencapsulated curcumin suppress beta-amyloid-induced cognitive impairments in rats: involvement of BDNF and Akt/GSK3beta signaling pathway. Neurobiol Learn Mem 106:134-144

57. Zeng Y, Zhao D, Xie CW (2010) Neurotrophins enhance CaMKII activity and rescue amyloid-beta-induced deficits in hippocampal synaptic plasticity. J Alzheimers Dis 21(3):823-831

58. Anderton BH, Callahan L, Coleman P, Davies P, Flood D, Jicha GA, Ohm T, Weaver C (1998) Dendritic changes in Alzheimer's disease and factors that may underlie these changes. Prog Neurobiol 55(6):595-609

59. Chai GS, Wang YY, Zhu D, Yasheng A, Zhao P (2017) Activation of beta2-adrenergic receptor promotes dendrite ramification and spine generation in APP/PS1 mice. Neurosci Lett 636:158-164

60. Ma QL, Yang F, Frautschy SA, Cole GM (2012) PAK in Alzheimer disease, Huntington disease and X-linked mental retardation. Cell Logist 2(2):117-125

61. Zhou F, Xie J, Zhang S, Yin G, Gao Y, Zhang Y, Bo D, Li Z, Liu $S$, Feng $C$ et al (2018) Lead, cadmium, arsenic, and mercury combined exposure disrupted synaptic homeostasis through activating the Snk-SPAR pathway. Ecotoxicol Environ Saf 163:674-684

62. Bamburg JR, Bernstein BW, Davis RC, Flynn KC, Goldsbury C, Jensen JR, Maloney MT, Marsden IT, Minamide LS, Pak CW et al (2010) ADF/Cofilin-actin rods in neurodegenerative diseases. Curr Alzheimer Res 7(3):241-250

Publisher's Note Springer Nature remains neutral with regard to jurisdictional claims in published maps and institutional affiliations. 